\title{
Superstatistics: Theoretical concepts and physical applications
}

\author{
Christian Beck \\ School of Mathematical Sciences, Queen Mary, University of London, \\ London E1 4NS, UK
}

\begin{abstract}
A review of the superstatistics concept is provided, including various recent applications to complex systems.
\end{abstract}

\section{Introduction}

Complex systems often exhibit a dynamics that can be regarded as a superposition of several dynamics on different time scales. As a very simple example consider e.g. a Brownian particle moving through a changing environment. Assume that the environment exhibits temperature fluctuations on a large scale. Then there is a relatively fast dynamics given by the velocity of the Brownian particle and a slow one given by the temperature changes of the environment, which is spatiotemporally inhomogeneous. The two effects produce a superposition of two statistics, or in a short, a 'superstatistics' [1, 2, 3, 4, 5, 6, 7, 8]. The concept of a superstatistics was introduced in [1], in the mean time many applications for a variety of complex systems have been pointed out 9 , 10, 11, 12, 13, 14, 15, 16, 17, 18, 19, 20, 21, 22, 23, 24]. The stationary probability distributions of superstatistical systems typically exhibit non-Gaussian behaviour with fat tails, which can decay e.g. with a power law, or as a stretched exponential, or in an even more complicated way.

Essential for the superstatistical approach is the existence of an intensive parameter $\beta$ which fluctuates on a large spatio-temporal scale. For the above simple example of a superstatistical Brownian particle, $\beta$ is the fluctuating inverse temperature of the environment, but in general $\beta$ can also be an effective friction constant, a changing mass parameter, a changing amplitude of Gaussian white noise, the 
fluctuating energy dissipation in turbulent flows, a fluctuating volatility in finance, an environmental parameter for biological systems, or simply a local variance parameter extracted from a signal. Some superstatistical models exhibit anomalous transport, others don't. Most superstatistical models are somewhat 'less anomalous' than Levy-type models, in the sense that usually more of the higher moments exist as compared to Levy processes. The tails of the distributions exhibit 'fat' tails, but usually these are less pronounced than for a Levy distribution.

The superstatistics concept is very general and has been applied to a variety of complex systems. Recent successful applications include hydrodynamic turbulence [9, 10, 2, 11, 12], pattern forming systems [14], cosmic rays [15], solar flares [13], mathematical finance [17, 18, 19], random matrix theory 20, networks [21], quantum systems at low temperatures [23, wind velocity fluctuations [16, 25], hydro-climatic fluctuations 22 and delay statistics in traffic models 24. The aim in the following is to explain the basic concepts and applications in an easy-going way.

\section{The basic idea}

Consider a complex system in a stationary nonequilibrium state that is driven by some external forces. Usually we think here of a physical system (e.g. a turbulent flow) but we may easily apply similar techniques to economic, biological, social systems, where the meaning of the mathematical variables will be different, though the mathematical structure is similar. Generally, a complex system will be inhomogeneous in space and in time. Effectively, it may consist of many spatial cells (or, the measured time series may consist of many time slices) where there are different values of some relevant system parameter $\beta$. The cell size is effectively determined by the correlation length of the continuously varying $\beta$-field. Superstatistical systems are characterized by a simplifying effect, namely the fact that the relaxation time is short so that each cell can be assumed to be in local equilibrium (in a certain approximation at least). Sometimes this property will be satisfied for a given complex system, sometimes not.

In the long-term run, the stationary distributions of the superstatistical inhomogeneous system arise as a superposition of Boltzmann

factors $e^{-\beta E}$ (or analogues of Boltzmann factors describing the local 
behaviour of the system under consideration) weighted with the probability density $f(\beta)$ to observe some value $\beta$ in a randomly chosen cell:

$$
p(E)=\int_{0}^{\infty} f(\beta) \frac{1}{Z(\beta)} \rho(E) e^{-\beta E} d \beta
$$

Here $E$ is an effective energy for each cell, $Z(\beta)$ is the normalization constant of $\rho(E) e^{-\beta E}$ for a given $\beta$, and $\rho(E)$ is the density of states.

A typical example is a Brownian particle of mass $m$ moving through a changing environment in $d$ dimensions. Such a Langevin model has applications in many different areas of science. In the simplest case we may write down a linear local Langevin equation for the velocity $\vec{v}$

$$
\dot{\vec{v}}=-\gamma \vec{v}+\sigma \vec{L}(t)
$$

$(\vec{L}(t)$ : $d$-dimensional Gaussian white noise) which becomes superstatistical due to the fact that for a fluctuating environment the parameter $\beta:=\frac{2}{m} \frac{\gamma}{\sigma^{2}}$ becomes a random varaible as well: It varies from cell to cell on a rather large spatio-temporal scale $T$ (this time scale $T$ should not be confused with the temperature, which is denoted as $\beta^{-1}$ ). Of course, for this example $E=\frac{1}{2} m v^{2}$, and while the local stationary distribution in each cell is Gaussian

$$
p(\vec{v} \mid \beta)=\left(\frac{\beta}{2 \pi}\right)^{d / 2} e^{-\frac{1}{2} \beta m v^{2}},
$$

the marginal distribution describing the long-time behaviour of the particle

$$
p(\vec{v})=\int_{0}^{\infty} f(\beta) p(\vec{v} \mid \beta) d \beta
$$

exhibits non-trivial behaviour. The large- $|v|$ tails of this distribution depend on the behaviour of $f(\beta)$ for $\beta \rightarrow 0$ [4]. As a result of the integration over $\beta$, the probability distribution $p(\vec{v})$ will typically have fat tails. These can be e.g. power law tails, stretched exponentials, or whatever.

One of the most important example for practical application is a $\chi^{2}$-distribution for $f(\beta)$ (see next section for concrete formulas) and a Gaussian distribution for $p(\vec{v} \mid \beta)$. In this case one obtains from eq. (4)

$$
p(\vec{v}) \sim \frac{1}{\left(1+(q-1) \frac{1}{2} b v^{2}\right)^{\frac{1}{q-1}}},
$$

where $q$ and $b$ are suitable parameters, and $v=|\vec{v}|$. The function on the right-hand side of eq. (5) is called a $q$-Gaussian [34] and denoted 
by $e_{q}^{-\frac{1}{2} b v^{2}}$. Note that $q$-Gaussians decay asymptotically with a power law for $v \rightarrow \infty$. They reduce to ordinary Gaussians for $q \rightarrow 1$. But power laws are not the only possibility one can get for superstatistical systems. Many examples will be discussed in the following sections.

A generalized thermodynamics for superstatistical systems has been recently developed in [8] (see also [5] for early attempts). Here one starts from a Boltzmann-Gibbs-Shannon entropy function that also includes contributions from the fluctuations in $\beta$. Given that entropy, one can do formally thermodynamics. Ordinary thermodynamics (no fluctuations in $\beta$ ) is contained as a special case in this more general formalism.

\section{Typical distributions $f(\beta)$}

The distribution $f(\beta)$ is determined by the spatio-temporal dynamics of the driven nonequilibrium system under consideration. By construction, $\beta$ is positive, so $f(\beta)$ cannot be a Gaussian. Let us here consider important examples of what to expect in typical experimental situations for driven nonequilibrium systems.

a) There may be many (nearly) independent microscopic random variables variables $\xi_{j}, j=1, \ldots, J$ contributing to $\beta$ in an additive way. For large $J$ their rescaled sum $\frac{1}{\sqrt{J}} \sum_{j=1}^{J} \xi_{j}$ will approach a Gaussian random variable $X_{1}$ due to the Central Limit Theorem (CLT). In total, there can be many subsystems consisting of such microscopic random variables, leading to $n$ Gaussian random variables $X_{1}, \ldots, X_{n}$ due to various degrees of freedom in the system. As mentioned before, $\beta$ needs to be positive and a positive $\beta$ is obtained by squaring these Gaussian random variables. The resulting $\beta=\sum_{i=1}^{N} X_{i}^{2}$ is $\chi^{2}$ distributed with degree $n$, i.e.

$$
f(\beta)=\frac{1}{\Gamma\left(\frac{n}{2}\right)}\left(\frac{n}{2 \beta_{0}}\right)^{n / 2} \beta^{n / 2-1} e^{-\frac{n \beta}{2 \beta_{0}}} .
$$

The marginal distributions obtained by integrating over all $\beta$ exhibit power-law tails for large enerfies $E$. They are $q$-exponentials, $p(E) \sim$ $e_{q}^{-b E}=(1+(q-1) b E)^{-1 /(q-1)}$, where $q$ and $b$ can be related to $n$ and $\beta_{0}[26,27$. Note that this statistics arises as a universal limit dynamics, i.e. the details of the microscopic random variables $\xi_{j}$ (e.g. their probability densities) are irrelevant. 
b) The same consideration as above may apply to the 'temperature' $\beta^{-1}$ rather than $\beta$ itself. $\beta^{-1}$ may the sum of several squared Gaussian random variables arising out of many microscopic degrees of freedom $\xi_{j}$. The resulting $f(\beta)$ is the inverse $\chi^{2}$-distribution given by

$$
f(\beta)=\frac{\beta_{0}}{\Gamma\left(\frac{n}{2}\right)}\left(\frac{n \beta_{0}}{2}\right)^{n / 2} \beta^{-n / 2-2} e^{-\frac{n \beta_{0}}{2 \beta}} .
$$

It generates distributions that have exponential decays in $\sqrt{E}[28$, 4, 29]. Again this superstatistics is universal: details of the $\xi_{j}$ are irrelevant.

c) Instead of $\beta$ being a sum of many contributions, for other systems (in particular turbulent ones) the random variable $\beta$ may be generated by multiplicative random processes. We may have a local cascade random variable $X_{1}=\prod_{j=1}^{J} \xi_{j}$, where $J$ is the number of cascade steps and the $\xi_{i}$ are positive microscopic random variables. Due to the CLT, $\log X_{1}=\sum_{j=1}^{J} \log \xi_{j}$ becomes Gaussian for large $J$ if it is properly rescaled. Hence $X_{1}$ is lognormally distributed. In general there may be $n$ such product contributions to the superstatistical varaiable $\beta, \beta=\prod_{i=1}^{n} X_{i}$. Then $\log \beta=\sum_{i=1}^{n} \log X_{i}$ is a sum of Gaussian random variables, hence it is Gaussian as well. Thus $\beta$ is lognormally distributed, i.e.

$$
f(\beta)=\frac{a}{\beta} \exp \left[-c(\ln \beta-b)^{2}\right],
$$

where $a, b, c$ are suitable constants. The result is independent of the details of the microscopic cascade random variables $\xi_{j}$, hence there is universality again. This type of lognormal superstatistics is particularly relevant for turbulent flows [12, 10, 9, 30, 11].

\section{Asymptotic behaviour for large en- ergies}

Superstatistical probability densities, as given by eq.(11) or (4), typically exhibit 'fat tails' for large $E$, but what is the precise functional form of this large energy behaviour? The answer depends on the distribution $f(\beta)$ and can be obtained from a variational principle. Details are described in [4], here we just summarize some results. For simplicity, let us put $\rho(E)=1$ in eq. (1). We may define a new probability 
density $\tilde{f}$ by

$$
\tilde{f}(\beta):=c \frac{f(\beta)}{Z(\beta)},
$$

where $c$ is a suitable normalization constant. The new density $\tilde{f}$ absorbes the $\beta$-dependence of the local partition function $Z(\beta)$. With this notation, $p(E)$ can now be regarded as the Laplace transform of $\tilde{f}$. Renaming $\tilde{f} \rightarrow f$ we obtain

$$
\begin{aligned}
p(E) & \sim \int_{0}^{\infty} f(\beta) e^{-\beta E} d \beta \\
& =\int_{0}^{\infty} e^{-\beta E+\ln f(\beta)} d \beta \\
& \sim e^{\sup _{\beta}\{-\beta E+\ln f(\beta)\}} \\
& =e^{-\beta_{E} E+\ln f\left(\beta_{E}\right)} \\
& =f\left(\beta_{E}\right) e^{-\beta_{E} E}
\end{aligned}
$$

Here we used the saddle point approximation. $\beta_{E}$ is the value of $\beta$ where the function $-\beta E+\ln f(\beta)$ has a maximum. The expression

$$
\sup _{\beta}\{-\beta E+\ln f(\beta)\}
$$

corresponds to a Legendre transform of $\ln f(\beta)$.

For the case where $f(\beta)$ is smooth and has only a single maximum we can obtain the supremum by differentiating, i.e.

$$
\sup _{\beta}\{-\beta E+\ln f(\beta)\}=-\beta_{E} E+\ln f\left(\beta_{E}\right)
$$

where $\beta_{E}$ satisfies the differential equation

$$
0=-E+(\ln f(\beta))^{\prime}=-E+\frac{f^{\prime}(\beta)}{f(\beta)} .
$$

By taking into account the next-order contributions around the maximum, eq. (10) can be improved to

$$
p(E) \sim \frac{f\left(\beta_{E}\right) e^{-\beta_{E} E}}{\sqrt{-\left(\ln f\left(\beta_{E}\right)\right)^{\prime \prime}}} .
$$

Let us consider a few examples. Consider an $f(\beta)$ which for small $\beta$ is of the power-law form $f(\beta) \sim \beta^{\gamma}, \gamma>0$. An example is the $\chi^{2}$ distribution of $n$ degrees of freedom, which was mentioned previously:

$$
f(\beta)=\frac{1}{\Gamma\left(\frac{n}{2}\right)}\left(\frac{n}{2 \beta_{0}}\right)^{n / 2} \beta^{n / 2-1} e^{-\frac{n \beta}{2 \beta_{0}}},
$$


$\left(\beta_{0} \geq 0, n>1\right)$. This behaves for $\beta \rightarrow 0$ as

$$
f(\beta) \sim \beta^{n / 2-1}
$$

i.e.

$$
\gamma=\frac{n}{2}-1
$$

Other examples exhibiting this power-law form are the so-called $F$ distributions [1, 29]. With the above formalism one obtains from eq. (13)

$$
\beta_{E}=\frac{\gamma}{E}
$$

and

$$
p(E) \sim E^{-\gamma-1} .
$$

These types of $f(\beta)$ form the basis for power-law generalized Boltzmann factors ( $q$-exponentials) used in generalized versions of statistical mechanics, so-called non-extensive statistical mechanics [31, 32, 33. 34. These depend on an entropic index $q$ coming from a more general entropy functional, and the relation between $\gamma$ and $q$ is

$$
\gamma+1=\frac{1}{q-1}
$$

Another example would be an $f(\beta)$ which for small $\beta$ behaves as $f(\beta) \sim e^{-c / \beta}, c>0$. In this case one obtains

$$
\beta_{E}=\sqrt{\frac{c}{E}}
$$

and

$$
p(E) \sim E^{-3 / 4} e^{-2 \sqrt{c E}} .
$$

The above example can be generalized to stretched exponentials: For $f(\beta)$ of the form $f(\beta) \sim e^{-c \beta^{\delta}}$ one obtains after a short calculation

$$
\beta_{E}=\left(\frac{E}{c|\delta|}\right)^{1 /(\delta-1)}
$$

and

$$
p(E) \sim E^{(2-\delta) /(2 \delta-2)} e^{a E^{\delta /(\delta-1)}},
$$

where $a$ is some factor depending on $\delta$ and $c$. In this case the superstatistical complex system exhibits stretched exponential tails. 


\section{Anomalous diffusion in superstatis- tical systems}

We now illustrate that superstatistical systems can exhibit normal as well as anomalous transport. This depends on the dynamical properties of the model considered.

For simplicity, we restrict ourselves to a 1-dimensional model. Let us again consider locally a 1-dimensional Brownian particle of mass $m$ and a Langevin equation of the form

$$
\dot{v}=-\gamma v+\sigma L(t)
$$

where $v$ denotes the velocity of the particle, and $L(t)$ is normalized Gaussian white noise with the following expectations:

$$
\begin{aligned}
\langle L(t)\rangle & =0 \\
\left\langle L(t) L\left(t^{\prime}\right)\right\rangle & =\delta\left(t-t^{\prime}\right) .
\end{aligned}
$$

We assume that the parameters $\sigma$ and $\gamma$ are constant for a sufficiently long time scale $T$, and then change to new values, either by an explicit time dependence, or by a change of the environment through which the Brownian particle moves. Formal identification with local equilibrium states in the spatial cells where $\beta$ is approximately constant (ordinary statistical mechanics at temperature $\beta^{-1}$ ) yields during the time scale $T$ the relation 35

$$
\left\langle v^{2}\right\rangle=\frac{\sigma^{2}}{2 \gamma}=\frac{1}{\beta m}
$$

or

$$
\beta=\frac{2}{m} \frac{\gamma}{\sigma^{2}} \text {. }
$$

Again, we emphasize that after the time scale $T, \gamma$ and $\sigma$ will take on

new values in a stochastic way. During a time interval of the order of $T$, the probability density $P(v, t)$ obeys the Fokker-Planck equation

$$
\frac{\partial P}{\partial t}=\gamma \frac{\partial(v P)}{\partial v}+\frac{1}{2} \sigma^{2} \frac{\partial^{2} P}{\partial v^{2}}
$$

with the local stationary solution

$$
P(v \mid \beta)=\sqrt{\frac{m \beta}{2 \pi}} \exp \left\{-\frac{1}{2} \beta m v^{2}\right\} .
$$


In the adiabatic approximation, valid for large $T$, one asumes that the local equilibrium state is reached very fast so that relaxation processes can be neglected. Within a cell in local equilibrium the correlation function is given by 35 .

$$
C\left(t-t^{\prime} \mid \beta\right)=\left\langle v(t) v\left(t^{\prime}\right)\right\rangle=\frac{1}{m \beta} e^{-\gamma\left|t-t^{\prime}\right|} .
$$

It is now interesting to see that the long-term invariant distribution $P(v)$, given by

$$
P(v)=\int_{0}^{\infty} f(\beta) P(v \mid \beta) d \beta
$$

depends only on the probability distribution of $\beta=\frac{2}{m} \frac{\gamma}{\sigma^{2}}$ and not on that of the single quantities $\gamma$ and $\sigma^{2}$. This means, one can obtain the same stationary distribution (33) from different dynamical models based on a Langevin equation with fluctuating parameters. Either $\gamma$ may fluctuate, and $\sigma^{2}$ is constant, or the other way round. On the other hand, the superstatistical correlation function

$$
C\left(t-t^{\prime}\right)=\int_{0}^{\infty} f(\beta) C\left(t-t^{\prime} \mid \beta\right) d \beta=\frac{1}{m} \int_{0}^{\infty} f(\beta) \beta^{-1} e^{-\gamma\left|t-t^{\prime}\right|} d \beta
$$

can distinguish between these two cases. The study of correlation functions thus yields more information for any superstatistical model.

Let us illustrate this with a simple example. Assume that $\sigma$ fluctuates and $\gamma$ is constant and that $\beta=\frac{2}{m} \frac{\gamma}{\sigma^{2}}$ is $\chi^{2}$-distributed. Since $\gamma$ is constant, we can move the exponential $e^{-\gamma\left|t-t^{\prime}\right|}$ out of the integral in eq. (34), meaning that the superstatistical correlation function still decays in an exponential way:

$$
C\left(t-t^{\prime}\right) \sim e^{-\gamma\left|t-t^{\prime}\right|}
$$

On the other hand, if $\sigma$ is constant and $\gamma$ fluctuates and $\beta$ is still $\chi^{2}$-distributed with degree $n$, we get a completely different answer. In this case, in the adiabatic approximation, the integration over $\beta$ yields a power-law decay of $C\left(t-t^{\prime}\right)$ :

$$
C\left(t-t^{\prime}\right) \sim\left|t-t^{\prime}\right|^{-\eta}
$$

where

$$
\eta=\frac{n}{2}-1
$$


Note that this decay rate is different from the asymptotic power law decay rate of the invariant density $P(v)$, which, using (31) and (33), is given by $P(v) \sim v^{-2 /(q-1)}$, with

$$
\frac{1}{q-1}=\frac{n}{2}+\frac{1}{2}
$$

Now let us proceed to the position

$$
x(t)=\int_{0}^{t} v\left(t^{\prime}\right) d t^{\prime}
$$

of the test particle. One has

$$
\left\langle x^{2}(t)\right\rangle=\int_{0}^{t} \int_{0}^{t}\left\langle v\left(t^{\prime}\right) v\left(t^{\prime \prime}\right)\right\rangle d t^{\prime} d t^{\prime \prime} .
$$

Asymptotic power-law velocity correlations with an exponent $\eta<1$ are expected to imply asymptotically anomalous diffusion of the form

$$
\left\langle x^{2}(t)\right\rangle \sim t^{\alpha}
$$

with

$$
\alpha=2-\eta \text {. }
$$

This relation simply results from the two time integrations.

It is interesting to compare our superstatistical model with other dynamical models generating anomalous diffusion. Plastino and Plastino 36 ] and Tsallis and Bukmann [37] study a generalized Fokker-Planck equation of the form

$$
\frac{\partial P(x, t)}{\partial t}=-\frac{\partial}{\partial x}(F(x) P(x, t))+D \frac{\partial^{2}}{\partial x^{2}} P(x, t)^{\nu}
$$

with a linear force $F(x)=k_{1}-k_{2} x$ and $\nu \neq 1$. Basically this model means that the diffusion constant becomes dependent on the probability density $P$. The probability densities generated by eq. (43) are $q$-Gaussians with the exponent

$$
q=2-\nu .
$$

The model generates anomalous diffusion with $\alpha=2 /(3-q)$. Assuming the validity of $\alpha=2-\hat{\eta}$, i.e. the generation of anomalous diffusion by slowly decaying velocity correlations with exponent $\hat{\eta}$, one obtains

$$
\hat{\eta}=\frac{4-2 q}{3-q} .
$$


On the other hand, for the $\chi^{2}$-superstatistical Langevin model one obtains by combining eq. (37) and (38) the different relation

$$
\eta=\frac{5-3 q}{2 q-2}
$$

Interesting enough, there is a distinguished $q$-value where both models yield the same answer:

$$
q=1.453 \Rightarrow \hat{\eta}=\eta=0.707
$$

These values of $q$ and $\eta$ correspond to realistic, experimentally observed numbers, for example in defect turbulence (see section 9).

\section{From time series to superstatistics}

We now want to be more practically orientated and apply superstatistical techniques to some complex systems (of whatever kind) where we do not know the equations of motion, and neither the distribution $f(\beta)$, but do have some information in form of a measured time series. Suppose an experimentally measured scalar time series $u(t)$ is given. Our goal is to test the hypothesis that it is due to a superstatistics and if yes, to extract $f(\beta)$. First we have to determine the superstatistical time scale $T$. For this we divide the time series into $N$ equal time intervals of size $\Delta t$. The total length of the signal is $t_{\max }=N \Delta t$. We then define a function $\kappa(\Delta t)$ by

$$
\kappa(\Delta t)=\frac{1}{t_{\max }-\Delta t} \int_{0}^{t_{\max }-\Delta t} d t_{0} \frac{\left\langle(u-\bar{u})^{4}\right\rangle_{t_{0}, \Delta t}}{\left\langle(u-\bar{u})^{2}\right\rangle_{t_{0}, \Delta t}^{2}}
$$

Here $\langle\cdots\rangle_{t_{0}, \Delta t}=\frac{1}{\Delta t} \int_{t_{0}}^{t_{0}+\Delta t} \cdots d t$ denotes an average over an interval of length $\Delta t$ starting at $t_{0}$. The integration result fluctuates for each value of $t_{0}$ and is averaged by the integral over $t_{0}$. $\bar{u}$ denotes the average of $u$. Now assume the simplest case, that our complex system dynamics basically arises out of a superposition of local Gaussians on some unknown time scale $T$. How can we extract $T$ ? We should be looking for the special value $\Delta t=T$ where

$$
\kappa(T)=3 .
$$

Clearly this condition defining the superstatistical time scale $T$ simply reflects the fact that we are looking for locally Gaussian behaviour in 


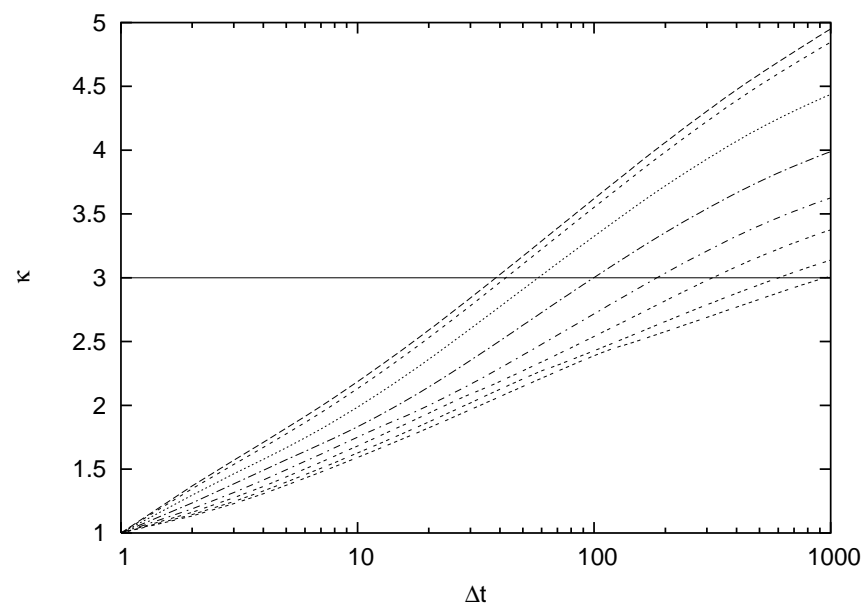

Figure 1: Determination of the superstatistical time scale $T$ from the intersection with the line $\kappa=3$ for turbulent Taylor-Couette flow, $\delta=2^{j}, j=$ $0,1,2, \ldots, 7$ (from top to bottom).

the time series, which implies a local flatness of 3 . If $\Delta t$ is so small that only one constant value of $u$ is observed in this interval, then of course $\kappa(\Delta t)=1$. On the other hand, if $\Delta t$ is so large that it includes the entire time series, then we obtain the flatness of the distribution of the entire signal, which is larger than 3 , since superstatistical distributions are generically fat-tailed. Inbetween, there should be a distinguished time scale where $\kappa=3$.

Fig. 1 shows the function $\kappa(\Delta t)$ for an example of a time series that has been studied in [2], the longitudinal velocity difference $u(t)=v(t+\delta)-v(t)$ in a turbulent Taylor-Couette flow on a scale $\delta$. For each scale $\delta$ the relevant superstatistical time scale $T$ leading to locally Gaussian behaviour can be extracted as the intersection with the line $\kappa=3$. These time scales $T$ are to be compared with the relaxation times $\gamma^{-1}$ of the dynamics, which can be estimated from the short-time exponential decay of the correlation function $\left.C\left(t-t^{\prime}\right)\right)=\left\langle u(t) u\left(t^{\prime}\right)\right\rangle$. One obtains the result that the ratio $T / \gamma^{-1}$ is pretty large and increases with Reynolds number [2]. This time scale separation is indeed the deeper reason why superstatistical models of turbulence work quite well [12, 2, 11].

Next, given a general signal $u(t)$ we are interested in the analysis of the slowly varying stochastic process $\beta(t)$. Since the variance of local 


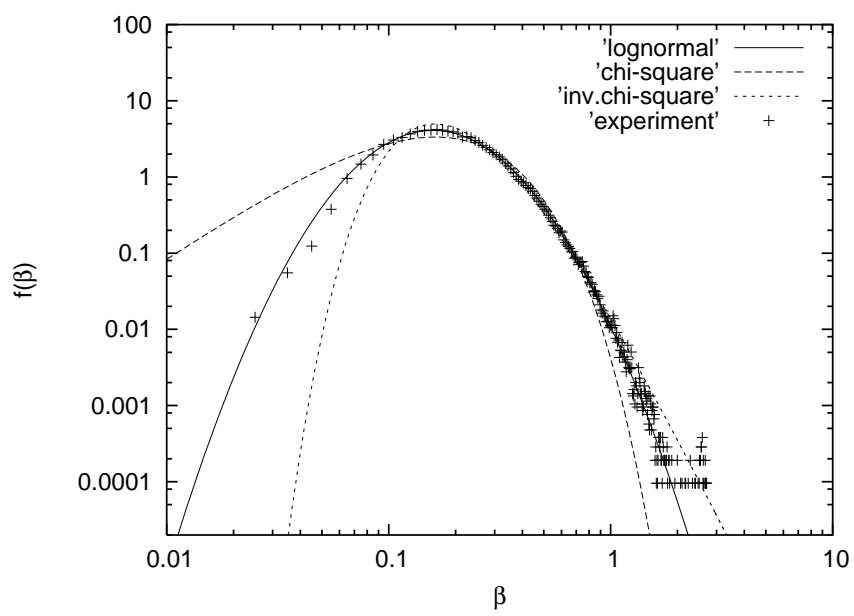

Figure 2: Probability distribution $f(\beta)$ as extracted from the measured turbulent time series in [2].

Gaussians $\sim e^{-\frac{1}{2} \beta u^{2}}$ is given by $\beta^{-1}$, we can determine the process $\beta(t)$ from the time series as

$$
\beta\left(t_{0}\right)=\frac{1}{\left\langle u^{2}\right\rangle_{t_{0}, T}-\langle u\rangle_{t_{0}, T}^{2}}
$$

We can then easily make a histogram of $\beta\left(t_{0}\right)$ for all values of $t_{0}$, thus obtaining the probability density $f(\beta)$.

Fig. 2 shows this probability density for our example of turbulent time series. Motivated by our consideration in section 3, the data are compared with a $\chi^{2}$-distribution, inverse $\chi^{2}$-distribution and lognormal distribution, all having the same mean $\langle\beta\rangle$ and variance $\left\langle\beta^{2}\right\rangle-\langle\beta\rangle^{2}$ as the experimental data. Clearly the lognormal distribution yields the best fit. Indeed, the cascade picture of energy dissipation in turbulent flow strongly suggests that lognormal superstatistics should be relevant, with $\beta$ being related to a suitable power of the fluctuating energy dissipation rate [27, 11]. However, again let us mention that other complex systems can generate a completely different type of superstatistics. Still the same methods apply.

For superstatistics to be a good approximation we need the variable $\beta(t)$ to change very slowly as compared to $u(t)$. This is indeed the case for our turbulence example, as can be seen in Fig. 3. 


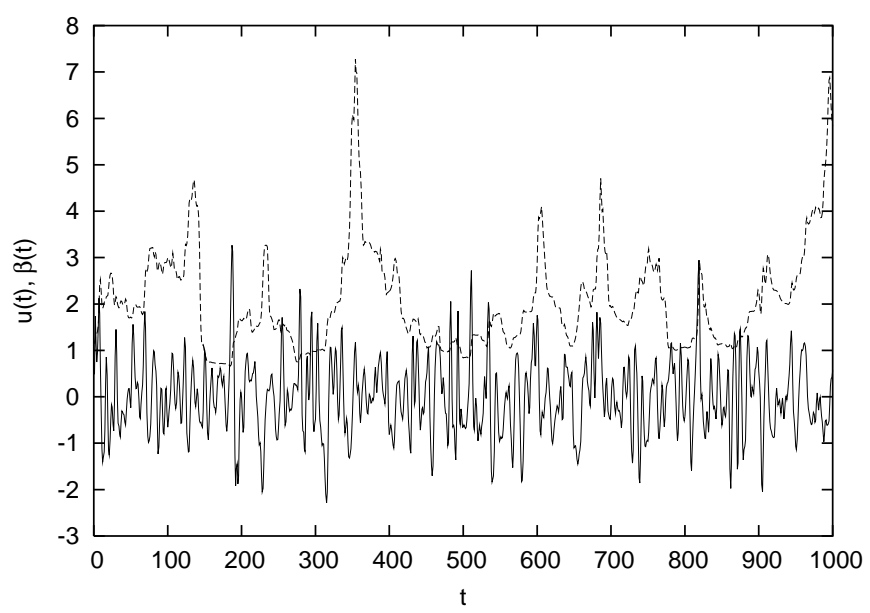

Figure 3: Typical evolution of $u(t)$ (solid line) and $\beta(t)$ (dashed line) for the turbulent Taylor-Couette flow.

\section{Overview of applications}

The superstatistics concept has been applied to many different complex systems. Let us first give a short overview of applications, after that we will treat four examples in more detail. Rizzo and Rapisarda [16, 25] study experimental data of wind velocities at Florence airport and find that superstatistics does a good job. Swinney et al. [2, 38] extract lognormal superstatistics for turbulent flow between counterrotating disks. Paczuski et al. [13] study data of solar flares on various time scales and embedd this into a superstatistical model based on $\chi^{2}$ superstatistics. Human behaviour when sending off print jobs might also stand in connection to such a superstatistics 39. Bodenschatz et al. [40, 41, 42] and Pinton et al. [43, 44] have detailed experimental data on the accelerations of single test particles in a turbulent flow, which are well described by lognormal superstatistics [9, 10, 11]. The statistics of cosmic rays is well described by $\chi^{2}$-superstatistics, with $n=3$ due to the three spatial dimensions [15]. In mathematical finance superstatistical techniques are well known and come under the heading 'volatility fluctuations', see e.g. [17, 18, 19]. Possible applications also include granular media, which could be described by different types of superstatistics, depending on the boundary conditions [45]. The observed fat tails of solar wind speed fluctuations 46] could also be related to a superstatistical model. Hydroclimatic fluctuations have 
been analysed using the superstatistics concept [22]. Briggs et al. 24] apply a superstatistical model to observed train delays on the British rail network. On the theoretical side, Chavanis $[6]$ points out analogies between superstatistics and the theory of violent relaxation for collisionless stellar systems. Abul-Magd [20] applies superstatistics to random matrix theory. Luczka and Zaborek [4] have studied a simple model of dichotomous fluctuations of $\beta$ on different time scales where everything can be calculated analytically. Mathai and Haubold [48 investigate a link between superstatistics and fractional reaction equations.

\section{Lagrangian turbulence}

We now treat some examples of applications in more detail. We start with the recent Lagrangian turbulence applications [11. Over the past few years there has been experimental progress [40, 41, 42, 43, 44] in tracking single test particles advected by a turbulent flow. This area of research is called 'Lagrangian turbulence'. To theoretically model Lagrangian turbulence, one may first start from a Gaussian turbulence model, the Sawford model [49, 50]. This model considers the joint stochastic process $(a(t), v(t), x(t))$ of an arbitrary component of acceleration, velocity and position of a Lagrangian test particle embedded in the turbulent flow, and assumes that they obey the stochastic differential equation

$$
\begin{aligned}
\dot{a}= & -\left(T_{L}^{-1}+t_{\eta}^{-1}\right) a-T_{L}^{-1} t_{\eta}^{-1} v \\
& +\sqrt{2 \sigma_{v}^{2}\left(T_{L}^{-1}+t_{\eta}^{-1}\right) T_{L}^{-1} t_{\eta}^{-1}} L(t) \\
\dot{v}= & a \\
\dot{x}= & v,
\end{aligned}
$$

where

$L(t)$ : Gaussian white noise

$T_{L}$ and $t_{\eta}$ : two time scales, with $T_{L}>>t_{\eta}$,

$T_{L}=2 \sigma_{v}^{2} /\left(C_{0} \bar{\epsilon}\right)$

$t_{\eta}=2 a_{0} \nu^{1 / 2} /\left(C_{0} \bar{\epsilon}^{1 / 2}\right)$

$\bar{\epsilon}$ : average energy dissipation

$C_{0}, a_{0}$ : Lagrangian structure function constants

$\sigma_{v}^{2}$ variance of the velocity distribution

$R_{\lambda}=\sqrt{15} \sigma_{v}^{2} / \sqrt{\nu \bar{\epsilon}}$ Taylor scale Reynolds number. 
For our purposes it is sufficient to consider the limit $T_{L} \rightarrow \infty$, which is a good approximation for large Reynolds numbers. In that limit the Sawford model reduces to just a linear Langevin equation for the acceleration

$$
\dot{a}=-\gamma a+\sigma L(t)
$$

with

$$
\begin{aligned}
\gamma & =\frac{C_{0}}{2 a_{0}} \nu^{-1 / 2} \bar{\epsilon}^{1 / 2} \\
\sigma & =\frac{C_{0}^{3 / 2}}{2 a_{0}} \nu^{-1 / 2} \bar{\epsilon}
\end{aligned}
$$

Note that this is a Langevin equation for the acceleration, so the meaning of the variables is slightly different as compared to the case of an ordinary Brownian particle, where the Langevin equation describes the velocity. In practice, the acceleration is measured as a velocity difference on a very small time scale.

Unfortunately, the Sawford model predicts Gaussian stationary distributions for $a$ and $v$, and is thus at variance with the recent measurements [41, 43], which provide evidence for distributions with fat tails. So how can we extend the Sawford model to make it physically realistic?

As said before, the idea is to generalize the Sawford model with constant parameters to a superstatistical Sawford model with fluctuating ones. To construct a superstatistical extension of Sawford model, one replaces the constant energy dissipation $\bar{\epsilon}$ by a fluctuating one. It is assumed to be lognormally distributed. Moreover, one extends the model to include all 3 components of the velocity and acceleration, as well as contributions from a fluctuating enstrophy (rotational energy) surrounding the test particle. From this new theory [11] excellent agreement with the experimental data is obtained, see Fig. 4 for an example. One obtains not only the correct 1-point probability distributions, but also good agreement for the decay of correlation

functions, the observed statistical dependencies between acceleration components, and scaling exponents.

\section{Defect turbulence}

Let us now consider another physically relevant example, so-called 'defect turbulence'. Defect turbulence shares with ordinary turbulence 


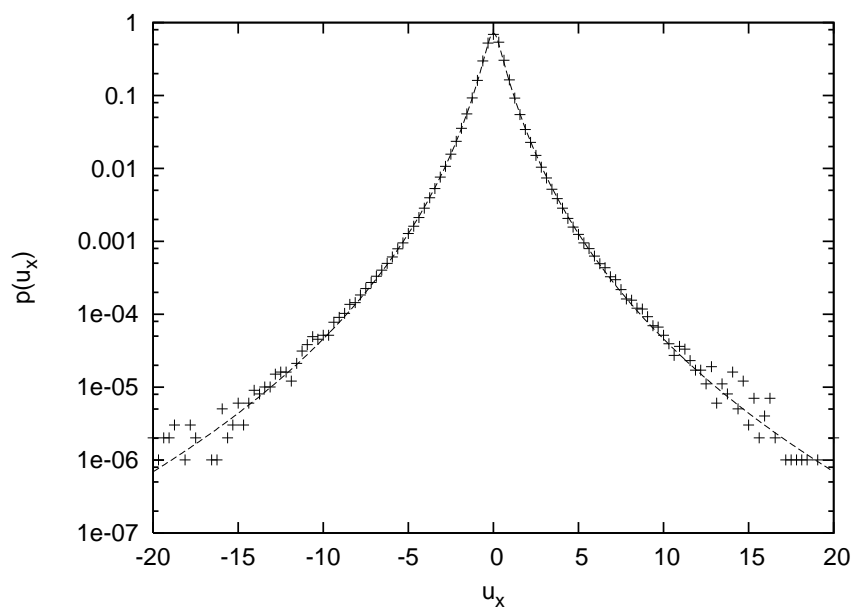

Figure 4: Predicted and measured probability density of a component of the small-scale velocity difference of a Lagrangian test particle in a turbulent flow. The dashed line is based on lognormal superstatistics [11]. The experimental data are from [43].

only the name, otherwise it is very different. It is a phenomenon related to convection and has nothing to do with fully developed hydrodynamic turbulence. Consider a Raleigh-Benard convection experiment: A liquid is heated from below and cooled from above. For large enough temperature differences, interesting convection patterns start to evolve. An inclined layer convection experiment [51, 52, 14, is a kind of Raleigh-Benard experiment where the apparatus is tilted by an angle (say 30 degrees), moreover the liquid is confined between two very narrow plates. For large temperature differences, the convection rolls evolve chaotically. Of particular interest are the defects in this pattern, i.e. points where two convection rolls merge into one (see Fig. 5). These defects behave very much like particles. They have a well-defined position and velocity, they are created and annihilated in pairs, and one can even formally attribute a 'charge' to them: There are positive and negative defects, as indicated by the black and white boxes in Fig. 5 .

The probability density of defect velocities has been quite precisely measured 14. As shown in Fig. 6, it quite precisely coincides with a $q$-Gaussian with $q \approx 1.46$. The defects are also observed to exhibit anomalous diffusion. Their position $X(t)$ roughly obeys an anomalous 


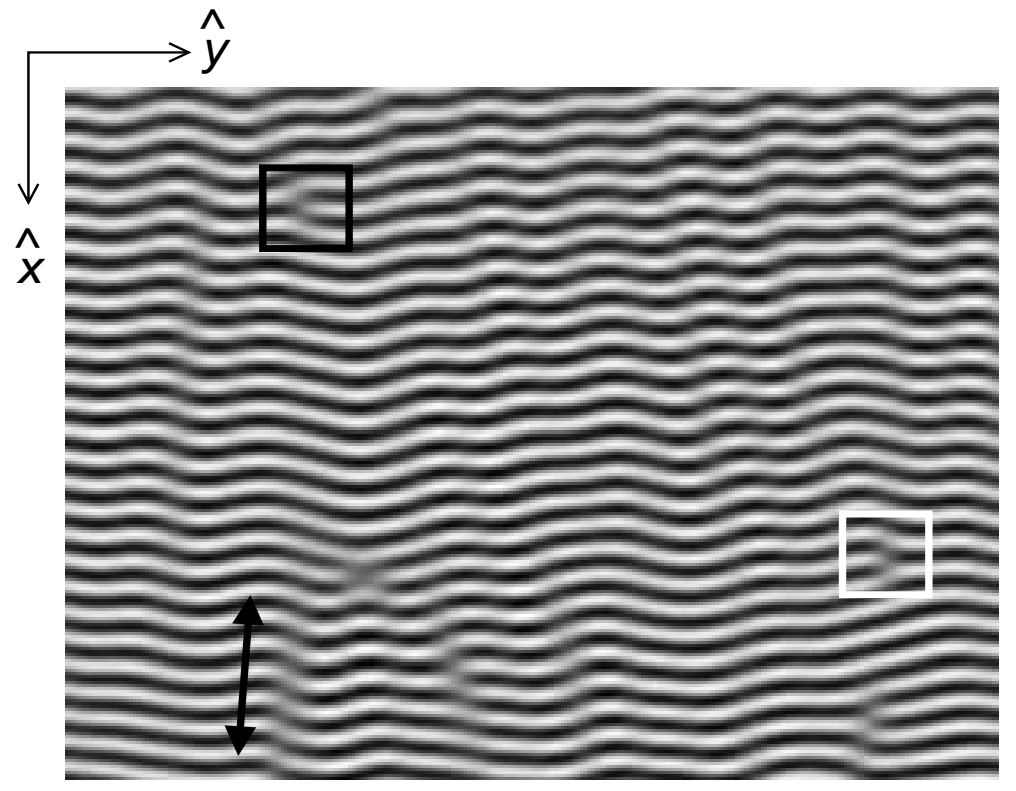

Figure 5: Convection rolls and defects (black and white boxes) as observed in the experiment of Daniels et al. [14].

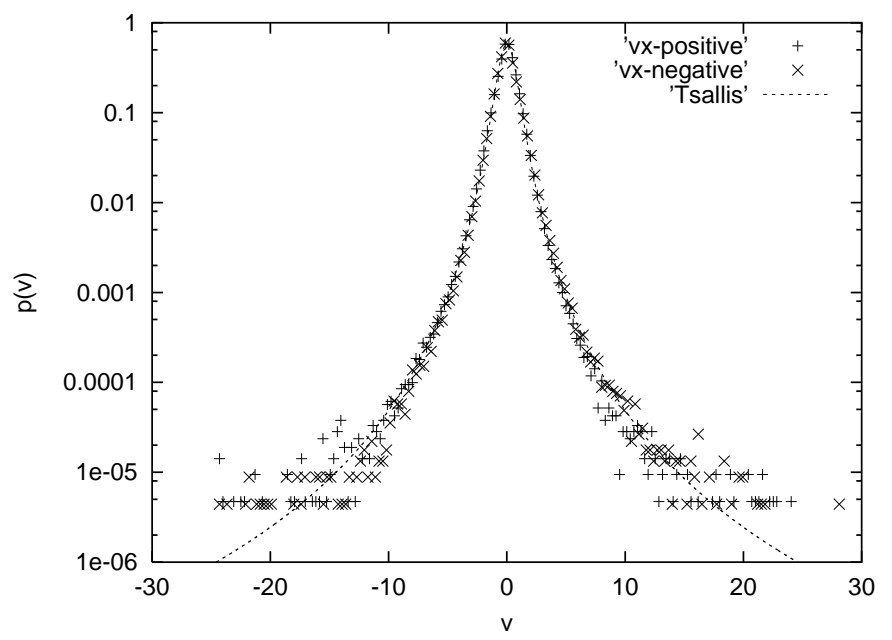

Figure 6: Measured probability density of defect velocities and fit with a $q$-Gaussian with $q=1.46$. 

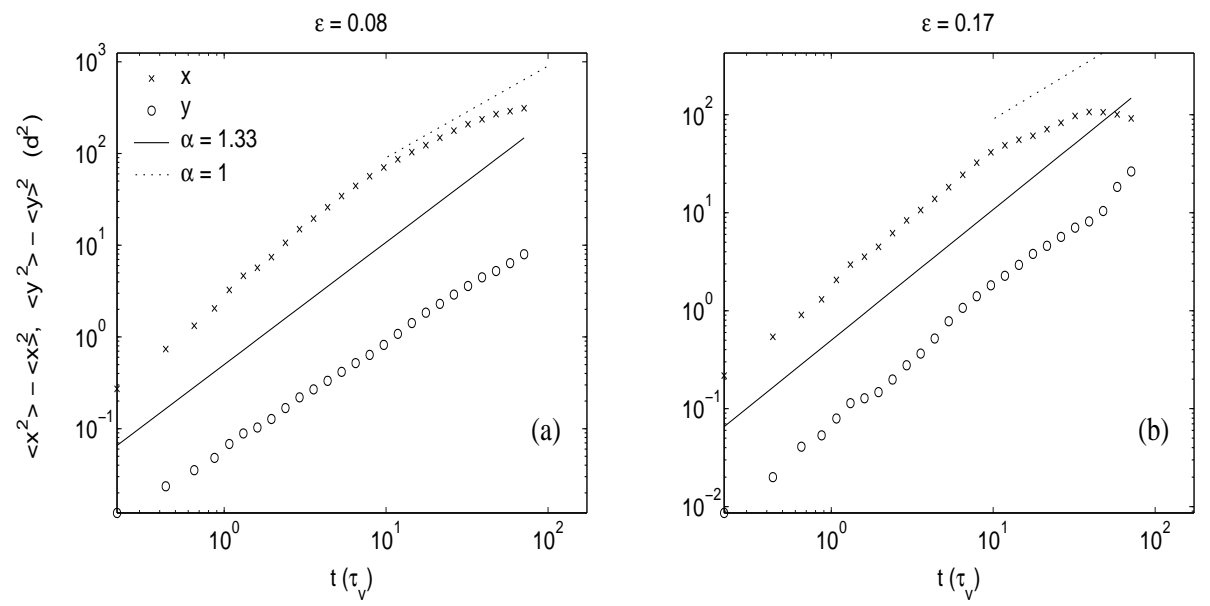

Figure 7: Measured anomalous diffusion of defects for two different values of the non-dimensional temperature differences $\epsilon$ between upper and lower plate.

diffusion law of the type

$$
\left\langle X^{2}(t)\right\rangle \sim t^{\alpha}
$$

where $\alpha \approx 1.33$ (see Fig. 7 ).

The simple superstatistical model of section 5 with fluctuating effective friction $\gamma$ makes sense as a very simple model for the defect velocity $v$. While ordinary Brownian particles have constant damping due to Stokes' law $\gamma=\frac{6 \pi \nu \rho a}{m}$, where $\nu$ is the kinematic viscosity of the liquid, $\rho$ is its density, $m$ is the mass of the particle and $a$ is the radius of the particle, defects are no ordinary particles: They are nonlinear excitations and have neither a well-defined mass $m$ nor a well-defined radius $a$. Thus one expects that there is an ensemble of damping constants $\gamma$ which depend on the topology of the defect and its fluctuating environment. In particular, the fastest velocities result from circumstances in which the defect is moving in a local environment with only a very small effective local damping $\gamma$ acting. The driving forces $L(t)$ are hardly damped during such a time interval, and lead to very large velocities for a limited amount of time, until another region with another $\gamma$ is reached. The result are $q$-Gaussians, as shown in Fig.6, and anomalous diffusion. In good approximation this system is described by $\chi^{2}$-superstatistics. 


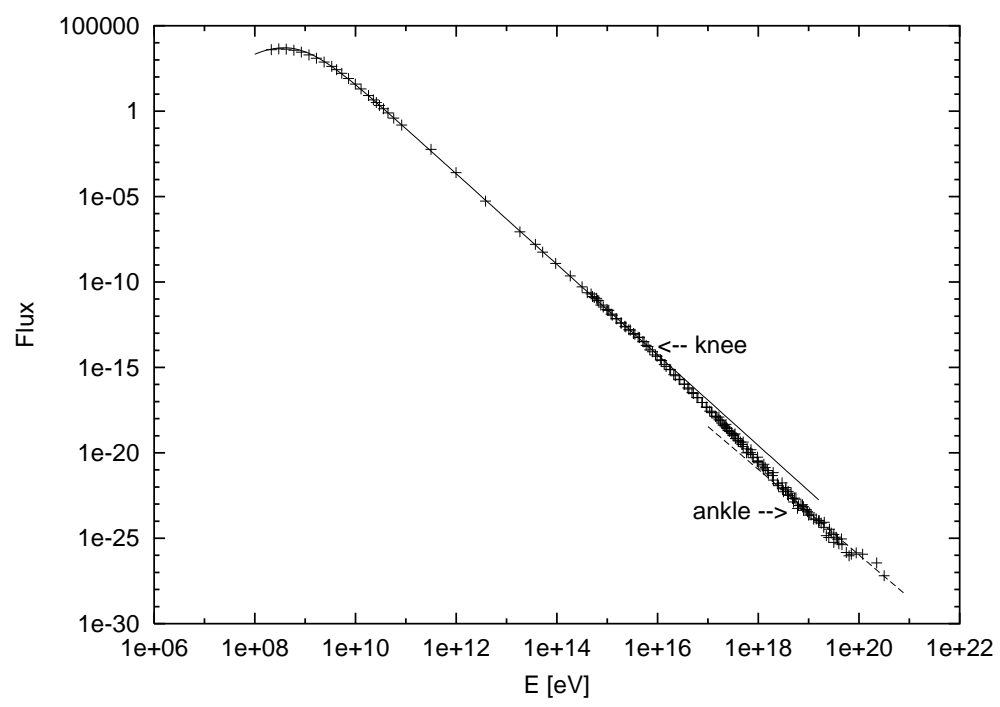

Figure 8: Observed energy spectrum of cosmic rays and a fit by eq. (58) with $q=1.215$.

\section{Statistics of cosmic rays}

Our third example is from high energy physics. We will proceed to extremely high temperatures, where (similar as in defect turbulence) particles are created and annihilated in pairs. We will apply superstatistical techniques to high energy collision processes on astrophysical scales, leading to the creation of cosmic ray particles that are ultimately observed on the earth. The idea to apply superstatistical techniques to the measured cosmic ray spectrum was first presented in [15], based on some earlier work in [53].

Experimental data of the measured cosmic ray energy spectrum are shown in Fig. 8. Also shown is a curve that corresponds to a prediction of a superstatistical model. Up to energies of $10^{16} \mathrm{eV}$, the measured flux rate of cosmic ray particles with a given energy $E$ is well fitted by a distribution of the form

$$
p(E)=C \cdot \frac{E^{2}}{(1+b(q-1) E)^{1 /(q-1)}} .
$$

$E$ is the energy of the particles,

$$
E=\sqrt{c^{2} p_{x}^{2}+c^{2} p_{y}^{2}+c^{2} p_{z}^{2}+m^{2} c^{4}}
$$


$b=(k \tilde{T})^{-1}$ is an effective inverse temperature variable, and $C$ is a constant representing the total flux rate. For relativistic particles the rest mass $m$ can be neglected and one has $E \approx c|\vec{p}|$. The distribution (58) is a $q$-generalized relativistic Maxwell-Boltzmann distribution in the formalism of nonextensive statistical mechanics [31. The factor $E^{2}$ takes into account the available phase space volume. As seen in Fig. 8, the cosmic ray spectrum is very well fitted by the distribution (58) if the entropic index $q$ is chosen as $q=1.215$ and if the effective temperature parameter is given by $k \tilde{T}=b^{-1}=107 \mathrm{MeV}$.

The above effective temperature is of the same order of magnitude as the so-called Hagedorn temperature $T_{H}$ [54, 55], an effective temperature well known from collider experiments. The Hagedorn temperature is much smaller than the center-of-mass energy $E_{C M S}$ of a typical collision process and represents a kind of 'boiling temperature' of nuclear matter at the confinement phase transition. It is a kind of maximum temperature that can be reached in a collision experiment. Even largest $E_{C M S}$ cannot produce a larger average temperature than $T_{H}$ due to the fact that the number of possible particle states grows exponentially.

Let us now work out the assumption that the power law of the measured cosmic ray spectrum is due to fluctuations of temperature. Assume that locally, in the creation process of some cosmic ray particle, some value of the fluctuating inverse temperature $\beta$ is given. We then expect the momentum of a randomly picked particle in this region to be distributed according to the relativistic Maxwell-Boltzmann distribution

$$
p(E \mid \beta)=\frac{1}{Z(\beta)} E^{2} e^{-\beta E} .
$$

Here $p(E \mid \beta)$ denotes the conditional probability of $E$ given some value of $\beta$. We neglect the rest mass $m$ so that $E=c|\vec{p}|$. The normalization constant is given by

$$
Z(\beta)=\int_{0}^{\infty} E^{2} e^{-\beta E} d E=\frac{2}{\beta^{3}} .
$$

Now assume that $\beta$ is $\chi^{2}$-distributed. The observed cosmic ray distribution at the earth does not contain any information on the local temperature at which the various particles were produced. Hence we have to average over all possible fluctuating temperatures, obtaining the measured energy spectrum as the marginal distribution

$$
p(E)=\int_{0}^{\infty} p(E \mid \beta) f(\beta) d \beta .
$$


The integral (62) with $f(\beta)$ given by (66) and $p(E \mid \beta)$ given by (60) is easily evaluated and one obtains eq. (58) with

$$
q=1+\frac{2}{n+6}
$$

and

$$
b=\frac{\beta_{0}}{4-3 q},
$$

where $\beta_{0}$ is the average inverse temperature.

The variables $X_{i}$ in section 3 describe the independent degrees of freedom contributing to the fluctuating temperature. At very large center of mass energies, due to the uncertainty relation, the probed volume $r^{3}$ is very small, and all relevant degrees of freedom in this small volume are basically represented by the 3 spatial dimensions into which heat can flow, leading to a fluctuating effective temperature in each creation process of cosmic ray particles. The 3 spatial degrees of freedom yield $n=3$ or, according to eq. (63),

$$
q=\frac{11}{9}=1.222 \text {. }
$$

For cosmic rays $E_{C M S}$ is very large, hence we expect a $q$-value that is close to this asymptotic value. The fit in Fig. 8 in fact uses $q=1.215$, which agrees with the predicted value in eq. (65) to about 3 digits.

\section{Statistics of train delays}

Our final example leaves the area of classical physics and turns to a more practical problem that almost everybody has experienced in the past. Trains, buses, planes, etc. are often delayed! A statistical analysis of train delay data in the UK was recently performed in [24]. One observes probability densities of delays that are $q$-exponentials, i.e. that have power-law tails (see Fig. 9 for an example).

We start with a very simple theoretical model for the local departure statistics of trains. The waiting time distribution until departure takes place is simply given by that of a Poisson process [35]

$$
P(t \mid \beta)=\beta e^{-\beta t} .
$$

Here $t$ is the time delay from the scheduled departure time, and $\beta$ is a positive parameter. The symbol $P(t \mid \beta)$ denotes the conditional 


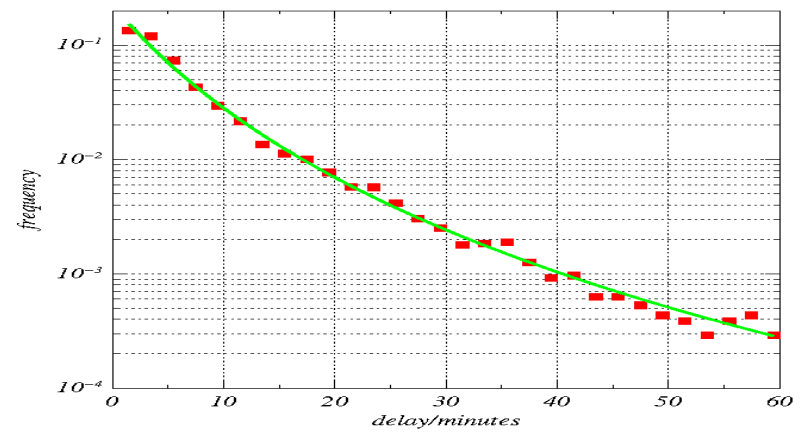

Figure 9: Delay statistics of train departures at Swindon station. The trains are heading for London Paddington.

probability density to observe the delay $t$ provided the parameter $\beta$ has a certain given value. Clearly, the above probability density is normalized. Large values of $\beta$ mean that most trains depart very well in time, whereas small $\beta$ describe a situation where long delays are rather frequent.

The above simple exponential model becomes superstatistical by making the parameter $\beta$ a fluctuating random variable as well. These fluctuations describe large-scale temporal variations of the British rail network environment. For example, during the start of the holiday season, when there is many passengers, we expect that $\beta$ is smaller than usual for a while, resulting in frequent delays. Similarly, if there is a problem with the track or if bad weather conditions exist, we also expect smaller values of $\beta$ on average. The value of $\beta$ is also be influenced by extreme events such as derailments, industrial action, terror alerts, etc.

The observed long-term distribution of train delays is then a mixture of exponential distributions where the parameter $\beta$ fluctuates. If $\beta$ is distributed with probability density $f(\beta)$, and fluctuates on a large time scale, then one obtains the marginal distributions of train 
delays as

$$
p(t)=\int_{0}^{\infty} f(\beta) p(t \mid \beta) d \beta=\int_{0}^{\infty} f(\beta) \beta e^{-\beta t} .
$$

Again, a $\chi^{2}$-distribution of $n$ degrees of freeedom makes sense for $\beta$, leading to $q$-exponential waiting time distributions of the form

$$
p(t) \sim(1+b(q-1) t)^{\frac{1}{1-q}}
$$

where $q=1+2 /(n+2)$ and $b=2 \beta_{0} /(2-q)$. Our model generates $q$-exponential distributions of train delays by a simple mechanism, namely a $\chi^{2}$-distributed parameter $\beta$ of the local Poisson process. This is in good agreement with the recorded delay data of the British rail network [24].

Typical $q$-values obtained from our fits for various stations are in the region $q=1.15 \ldots 1.35$ (see Table 1 ). Hence

$$
n=\frac{2}{q-1}-2
$$

is in the region $4 \ldots 11$. This means the number of degrees of freedom influencing the value of $\beta$ is just of the order we expected it to be: $A$ few large-scale phenomena such as weather, seasonal effects, passenger fluctuations, signal failures, repairs of track, etc. seem to be relevant. In general, it makes sense to compare stations with the same $q$ (the same number of external degrees of freedom of the network environment): The larger the value of $b$, the better the performance of this station under the given environmental conditions.

\section{References}

[1] C. Beck and E.G.D. Cohen, Physica A 322, 267 (2003)

[2] C. Beck, E.G.D. Cohen, and H.L. Swinney, Phys. Rev. E 72, 026304 (2005)

[3] C. Beck and E.G.D. Cohen, Physica A 344, 393 (2004)

[4] H. Touchette and C. Beck, Phys. Rev. E 71, 016131 (2005)

[5] C. Tsallis and A.M.C. Souza, Phys. Rev. E 67, 026106 (2003)

[6] P.-H. Chavanis, Physica A 359, 177 (2006) 
[7] C. Vignat, A. Plastino and A.R. Plastino, cond-mat/0505580

[8] S. Abe, C. Beck, and E.G.D. Cohen, arXiv:0705.0148 [cond-mat]

[9] C. Beck, Europhys. Lett. 64, 151 (2003)

[10] A. Reynolds, Phys. Rev. Lett. 91, 084503 (2003)

[11] C. Beck, Phys. Rev. Lett. 98, 064502 (2007)

[12] C. Beck, Physica D 193, 195 (2004)

[13] M. Baiesi, M. Paczuski and A.L. Stella, Phys. Rev. Lett. 96, 051103 (2006)

[14] K. E. Daniels, C. Beck, and E. Bodenschatz, Physica D 193, 208 (2004)

[15] C. Beck, Physica A 331, 173 (2004)

[16] S. Rizzo and A. Rapisarda, Environmental atmospheric at Florence airport, Proceedings of the 8th Experimental Chaos Conference, Florence, AIP Conf. Proc. 742, 176 (2004) (cond-mat/0406684)

[17] J.-P. Bouchard and M. Potters, Theory of Financial Risk and Derivative Pricing, Cambridge University Press, Cambridge (2003)

[18] M. Ausloos and K. Ivanova, Phys. Rev. E 68, 046122 (2003)

[19] Y. Ohtaki and H.H. Hasegawa, cond-mat/0312568

[20] A.Y. Abul-Magd, Physica A 361, 41 (2006)

[21] S. Abe and S. Thurner, Phys. Rev. E 72, 036102 (2005)

[22] A. Porporato, G. Vico, and P.A. Fay, Geophys. Res. Lett. 33, L15402 (2006)

[23] A.K. Rajagopal, cond-mat/0608679

[24] K. Briggs and C. Beck, Physica A 278, 498 (2007)

[25] S. Rizzo and A. Rapisarda, Application of superstatistics to atmospheric turbulence, in Complexity, Metastability and Nonextensivity, eds. C. Beck, G. Benedek, A. Rapisarda, C. Tsallis, World Scientific (2005) cond-mat/0502305

[26] G. Wilk and Z. Wlodarczyk, Phys. Rev. Lett. 84, 2770 (2000)

[27] C. Beck, Phys. Rev. Lett. 87, 180601 (2001) 
[28] H. Touchette, Temperature fluctuations and mixtures of equilibrium states in the canonical ensemble, in M. Gell-Mann, C. Tsallis (Eds.), Nonextensive Entropy - Interdisciplinary Applications, Oxford University Press (2004)

[29] F. Sattin and L. Salasnich, Phys. Rev. E 65, 035106(R) (2002)

[30] B. Castaing, Y. Gagne, and E.J. Hopfinger, Physica D 46, 177 (1990)

[31] C. Tsallis, J. Stat. Phys. 52, 479 (1988)

[32] C. Tsallis, R.S. Mendes and A.R. Plastino, Physica A 261,

[33] C. Tsallis, Braz. J. Phys. 29, 1 (1999)

[34] S. Abe, Y. Okamoto (eds.), Nonextensive Statistical Mechanics and Its Applications, Springer, Berlin (2001)

[35] N.G. van Kampen, Stochastic Processes in Physics and Chemistry, North Holland, Amsterdam (1981)

[36] A.R. Plastino and A. Plastino, Physica 222A, 347 (1995)

[37] C. Tsallis and D.J. Bukmann, Phys. Rev. 54E, R2197 (1996)

[38] S. Jung and H.L. Swinney, Phys. Rev. E 72, 026304 (2005)

[39] U. Harder and M. Paczuski, cs/PF/0412027

[40] A. La Porta, G.A. Voth, A.M. Crawford, J. Alexander, and E. Bodenschatz, Nature 409, 1017 (2001)

[41] N. Mordant, A.M. Crawford, and E. Bodenschatz, Phys. Rev. Lett. 93, 214501 (2004)

[42] A.M. Reynolds, N. Mordant, A.M. Crawford, and E. Bodenschatz, New Journal of Physics 7, 58 (2005)

[43] N. Mordant, P. Metz, O. Michel, and J.-F. Pinton, Phys. Rev. Lett 87, 214501 (2001)

[44] N. Mordant, E. Leveque, and J.-F. Pinton, New Journal of Physics 6, 116 (2004)

[45] J.S. van Zon et al., cond-mat/0405044

[46] L.F. Burlaga and A.F. Vinas, J. Geophys. Res. 109, A12107 (2004)

[47] J. Luczka and B. Zaborek, Acta Phys. Polon. B 35, 2151 (2004)

[48] A.M. Mathai and H.J. Haubold, Physica A 375, 110 (2007) 
[49] B.L. Sawford, Phys. Fluids A3, 1577 (1991)

[50] S.B. Pope, Phys. Fluids 14, 2360 (2002)

[51] K. E. Daniels, E. Bodenschatz, Phys. Rev. Lett. 88, 034501 (2002)

[52] K. E. Daniels, E. Bodenschatz, Chaos 13, 55 (2003)

[53] C. Tsallis, J.C. Anjos, E.P. Borges, Phys. Lett. A 310, 372 (2003)

[54] R. Hagedorn, Nuovo Cimento 3, 147 (1965)

[55] C. Beck, Physica A 286, 164 (2000) 


\begin{tabular}{lccl}
\hline station & $q$ & $b$ & code \\
\hline Bath Spa & 1.195 & 0.209 & BTH \\
Birmingham & 1.257 & 0.271 & BHM \\
Cambridge & 1.270 & 0.396 & CBG \\
Canterbury East & 1.298 & 0.400 & CBE \\
Canterbury West & 1.267 & 0.402 & CBW \\
City Thameslink & 1.124 & 0.277 & CTK \\
Colchester & 1.222 & 0.272 & COL \\
Coventry & 1.291 & 0.330 & COV \\
Doncaster & 1.289 & 0.332 & DON \\
Edinburgh & 1.228 & 0.401 & EDB \\
Ely & 1.316 & 0.393 & ELY \\
Ipswich & 1.291 & 0.333 & IPS \\
Leeds & 1.247 & 0.273 & LDS \\
Leicester & 1.231 & 0.337 & LEI \\
Manchester Piccadilly & 1.231 & 0.332 & MAN \\
Newcastle & 1.378 & 0.330 & NCL \\
Nottingham & 1.166 & 0.209 & NOT \\
Oxford & 1.046 & 0.141 & OXF \\
Peterborough & 1.232 & 0.201 & PBO \\
Reading & 1.251 & 0.268 & RDG \\
Sheffield & 1.316 & 0.335 & SHF \\
Swindon & 1.226 & 0.253 & SWI \\
York & 1.311 & 0.259 & YRK
\end{tabular}

Table 1: The fitted parameters $q$ and $b$ for the departure statistics of $23 \mathrm{UK}$ stations. 